\title{
Microwave testing of moist and oven-dry wood to evaluate grain angle, density, moisture content and the dielectric constant of spruce from $8 \mathrm{GHz}$ to $12 \mathrm{GHz}$
}

\author{
Andreas Aichholzer ${ }^{1,2} \cdot$ Christian Schuberth $^{2} \cdot$ Herwig Mayer $^{1} \cdot$ Holger Arthaber ${ }^{2}$
}

Received: 4 February 2016 / Published online: 30 May 2017

(C) The Author(s) 2017. This article is an open access publication

\begin{abstract}
The scope of this work is to discuss the challenges and demonstrate the potential of microwave testing for applications in the wood processing industry. Microwave technology benefits from the anisotropic dielectric properties of wood to simultaneously identify grain angle, density, and moisture content of wood. Therefore, the theory of free space transmission measurement is thoroughly discussed with emphasis on the characteristics of (and how to deal with) reflections occurring in real measurements. A more sophisticated calculation method for the derivation of the desired physical wood properties is presented. The advantages of a modern laboratory style setup are shown and its possible transition in an industrial-style application is discussed. Moist (moisture content 7.6-14\%) and oven-dry spruce samples are tested. The detection of grain angle for moist and oven-dry wood yields an RMSE (rootmean-squared-error) of $0.14^{\circ}$ and $0.4^{\circ}$, respectively. Moisture content is evaluated with density- and thickness-independent methods. Adapted regression models are proposed yielding an RMSE for moisture content of $0.45 \%$ for a single frequency measurement. The promising advantages of wood moisture estimation with frequency sweeps instead of fixed frequency signals are discussed and demonstrated for all samples (RMSE 0.39\%). The dielectric constant of moist and oven dry spruce in the range from 8 to $12 \mathrm{GHz}$ is evaluated in respect to density, moisture content and
\end{abstract}

Andreas Aichholzer

an.aichholzer@gmail.com

1 Institute of Physics and Materials Science, University of Natural Resources and Life Sciences, Peter-Jordan-Street 82, 1190 Vienna, Austria

2 Institute of Electrodynamics, Microwave and Circuit Engineering, Vienna University of Technology, Gußhausstr. 25/354, 1040 Vienna, Austria temperature. The respective constants $\varepsilon^{\prime}, \varepsilon^{\prime \prime}$, and $\tan (\delta)$ are formulated in a general form via a non-linear regression and compared to existing data in literature.

\section{Introduction}

Non-destructive evaluation of the physical properties of wood is a key task in modern wood manufacturing processes. Due to a rapidly increasing production speed, fast, non-contacting, reliable and robust measurement systems are demanded by the wood industry. When wood is used as a construction material, grain angle deviation, moisture content, and density are crucial parameters. Moisture is commonly determined with capacitance type moisture meters using high frequency signals or with resistance type pin moisture meters (Wilson 1999). These moisture meters are able to reasonably cope with industrial needs. However, due to their measuring principles, measurements are prone to deviations in the wood grain angle. Moreover, they require density information for a reliable moisture determination. The application of X-rays is successfully established for density measurements enhancing both production speed and quality (Schajer 2001). Still, these systems remain rather costly and require efforts for the protection against hazardous ionizing radiation. Grain angle deviations, both on a local (knot, top rupture) and global (sweep, taper, spiral growth) scale, significantly influence the strength of wood (Kollmann and Côté 1984). Consequently, production standards define boundary values for grain deviation for the application of solid wood in products as glued laminated timber [EN 1912 (2012)]. With modern drying technologies and planing, the occurrence of drying cracks has virtually been eliminated. Thus, global grain deviations remain undetected by conventional visual grading. The use 
of the tracheid-effect, i.e. the extension of a circular light spot to an elliptical form with the major axis oriented in grain direction (Nyström 2003) was discussed and applied to industrial applications. However, due to its measuring principle, this method demands clean and planed surfaces of the tested specimen. Moreover, this method is only about testing the surface and features deficient accuracy. A fast, robust, and accurate measurement device for grain angle detection in industrial conditions still is a considerable challenge for research.

Through the years, microwaves have consistently been demonstrated as a promising tool to reveal the key parameters density, moisture content, and grain deviation in a fast, non-destructive and contactless manner (King and Yen 1981). Several attempts are reported in literature to measure moisture and density of wood with microwaves (Al-Mattarneh et al. 2001; Johansson et al. 2003; Lundgren et al. 2006; Martin et al. 1987; Tiuri and Heikkila 1979). However, the lack of grain angle information makes these measurements prone to deviations in the grain direction. An early attempt to detect wood fiber orientation with a microwave resonator was done in 1974 (Tiuri and Liimatainen 1974). A grain angle detection using transmission measurements was presented by several authors (Leicester and Seath 1996; Malik et al. 2005; Shen et al. 1994). An integral approach using the microwave technology is able to determine density, moisture content, and grain angle simultaneously (Heikkila et al. 1982; James et al. 1985; Schajer and Orhan 2006). Here, the method intrinsically accounts for the influence of all three parameters and determines every parameter separately. Phase-shift and attenuation measurements yield density and moisture content via a multi-variant statistical approach, and according to depolarization, the grain angle is obtained. While Schajer and Orhan (2006) used a measurement setup featuring a scattering dipole between the antennas, recently reported setups (Aichholzer et al. 2013; Bogosanovic et al. 2011; Denzler et al. 2013; Vallejos and Grote 2009) benefit from superior dual-linear-polarized designs. The theoretical formulation of all these works directly follows Schajer and Orhan (2005). Schajer and Orhan, however, pointed out that they are measuring only a so-called "effective" transmission coefficient by ignoring any kind of occurring reflections of the testing signal. Moreover, no author accounted for the complex attenuations occurring in the sample-air-interfaces.

Strong influence of antenna design on the quality and applicability of microwave testing was encountered (Bogosanovic et al. 2013; Denzler et al. 2014; Vikberg et al. 2012a) and evaluated (Vikberg et al. 2012b). All these authors reported remarkable influence of diffraction at the sample edges that severely compromises testing quality. Bogosanovic et al. (2013) concretize, for their focused beam setup, that diffraction effects at the edges of the sample are negligible only if the minimum transverse dimension of the sample is greater than three times the width of the illuminating testing beam. Consequently, for lumber dimension $100 \times 500 \mathrm{~mm}$, they applied additional absorbers to prevent diffraction effects.

Up to now, no industrial application of microwave testing could be sustainably established in the wood manufacturing industry. This is explained on the one hand by the above addressed, challenging physical nature of microwaves and on the other hand by a lack of appropriate, low-cost, and robust electronic equipment. All previous work is based on several assumptions and simplifications without submitting exact theoretical evidence that these measures are justified. In this paper, the most promising method for free-space transmission measurements is discussed and new developments are introduced. For the first time in wood testing literature, a complete and theoretical derivation for the transmission coefficients is presented that addresses all, until now, unjustified assumptions and applies superior complex matrix calculation. With versatile laboratory equipment, the method's high potential is demonstrated and an outlook on obvious and near future applications is given.

\section{Theory}

\subsection{Microwave propagation in wood}

Microwaves propagating through a device under test (DUT) are attenuated and phase-shifted. For wood as DUT, both attenuation and phase shift are different in the three anatomic directions. The axial direction is parallel $(\|)$ to the wood grain. Radial and tangential directions show similar dielectric behavior and are both considered as a single "perpendicular" $(\perp)$ direction (Torgovnikov 1993). This idealized orthotropic wood model proved to be a sufficient approximation. Moreover, this simplification uniformly describes all possible growth-ring arrangements from quarter sawn to flat sawn.

Consequently, the dielectric properties of wood as an orthotropic material exhibit an elliptical character with the major (!) axis in grain direction (Fig. 1a). An incident linearly polarized test signal, with arbitrary polarization angle, can be decomposed into a component parallel and perpendicular to grain direction, respectively. These two components are independent waves propagating through wood with different attenuation and speed, i.e. phase shift. The resulting signal after the transmission through wood is the superposition of both components. In the general case, this resulting signal is elliptically polarized with the main axis rotated relative to the 
(a)
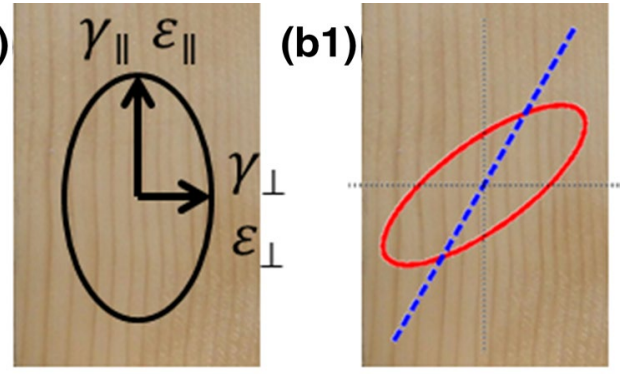

(b2)

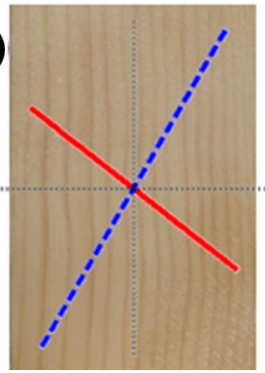

(b3)

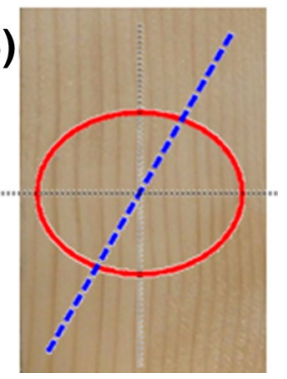

Fig. 1 a Elliptic character of the dielectric properties of wood (the propagation constant $\gamma$ and the dielectric constant $\varepsilon$ are introduced in Eqs. (2) and (6), respectively]. b1-b3 For a linearly polarized incident signal (dashed line, polarization plane $30^{\circ}$ off grain direction) possible signals (solid line) after transmission through wood are shown

grain direction. Figure 1b1-b3 shows possibly measured signals for typical wood attenuation ratio $\left(\mathrm{att}_{\|} / \mathrm{att}_{\perp}=2.2\right)$ and several phase shift differences of parallel and perpendicular signal. The general case in Fig. $1 \mathrm{~b} 1$ is demonstrated exemplarily with a phase shift difference of $320^{\circ}$. Only in special constellations, i.e. phase shift difference $0^{\circ} / 180^{\circ}$ or $90^{\circ} / 270^{\circ}$, linear polarization (Fig. $1 \mathrm{~b} 2$ for the case of $180^{\circ}$ ) or elliptical polarization [with minor (!) axis in grain direction, Fig. 1b3] of the resulting signal is possible.

Even in recent publications (e.g. Denzler and Weidenhiller 2015) this interrelation is misunderstood and illustrated incorrectly. However, exactly this behavior of wood due to its physical properties justifies and mandatorily requires a dual-linear-polarized measurement setup. Consider a detected signal that is measured in the polarization plane of the incident signal: Fig. 1b1-b3 demonstrates that signal detection in a single polarization plane is heavily prone to grain angle deviation. As a consequence, setups measuring in one polarization plane only (Johansson et al. 2003; Leicester and Seath 1996) are always prone to systematic errors. The same holds for setups with a single, elliptically or circularly polarized testing signal. Here, the measurement of a single polarization state comprises an unresolvable superposition of two otherwise subsequently performed linear polarized measurements.
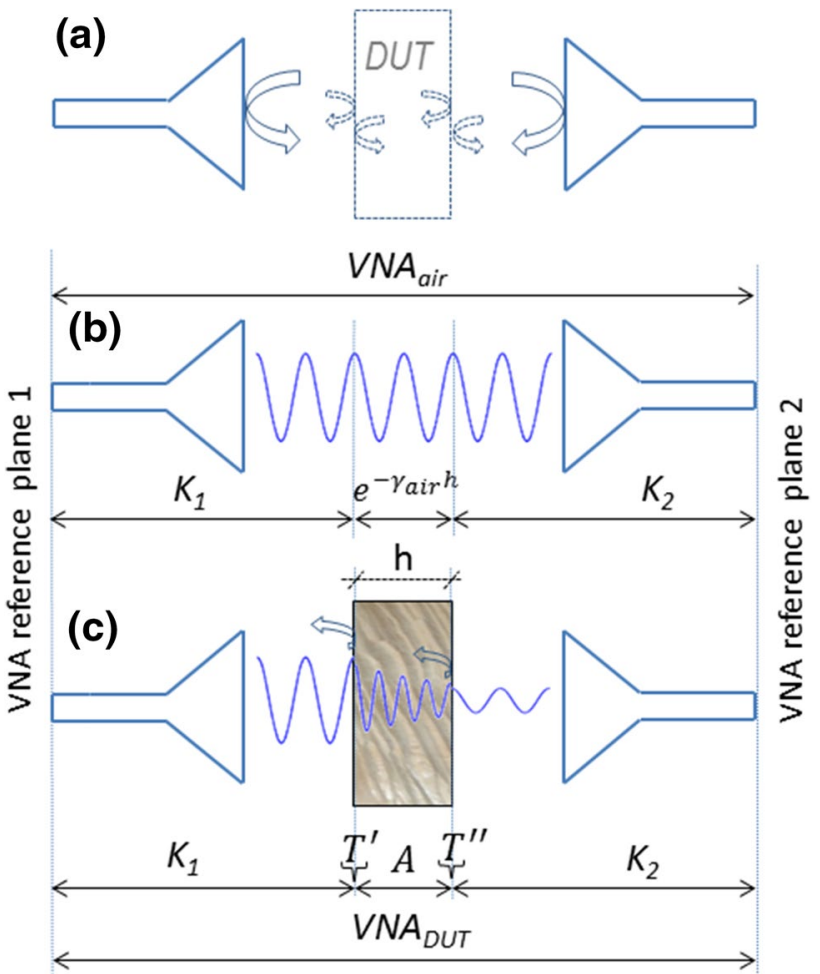

Fig. 2 Schematic of free-space transmission measurement. a Reflections occurring in real measurements. b Measurement through air. $\mathbf{c}$ Measurement of a DUT

However, the demonstration in Fig. 1 still is an idealized model as multiple reflections that occur within the sample are not yet considered.

\subsection{Reflections}

In actual measurements, reflections occur in each plane, where the characteristic wave impedance changes (Fig. 2a) (Kark 2006). Thus, a measurement solely through air features reflections at the antennas (solid arrows in Fig. 2a) which result in multiple reflections. This undesired effect can be weakened by the use of absorbing material between the antennas (Zhang and Okamura 1999) or compensated by the use of time-gating (Agilent Technologies 2007). With a DUT in place, additional reflections occur every time a signal passes a DUT/air-interface (dashed arrows in Fig. 2a). On the one hand, it yields subsequent reflections at the antennas and therefore a virtual additional testing signal that deteriorates the measurement quality. On the other hand, it results in multiple reflections in the sample. Multiple reflections generate a secondary signal that interferes with the direct path. For thicknesses of the DUT corresponding to half the wavelength of the testing signal in the DUT (and multiples thereof) this secondary signal even yields constructive interference and, therefore, less 
measured attenuation, which was shown by Robertson and Buckmaster (1992) on glass and plywood. Most work in the field neglected this effect, which is a sufficient assumption for wood at moisture contents $(<30 \%)$ and thicknesses $(>3 \mathrm{~cm})$ at interest. As a rule of thumb $10 \mathrm{~dB}$ attenuation of the secondary signal is sufficient. The effect vanishes with increasing thickness of the DUT. However, with the use of time gating (Agilent Technologies 2007; Kraszewski et al. 2001) applied in this work, only the direct path is measured. In this case, the only remaining two reflections to be accounted for in a measurement occur at both DUT/ air-interfaces.

For wood as DUT, multiple reflections occur for parallel and perpendicular path independently. Theoretically, this may even lead to positive interference in the II-path and negative interference in the $\perp$-path at the same time and hence to a stronger, i.e. less attenuated signal in parallel direction. For the typical range of wood properties ( $\mathrm{att}_{\|} / \mathrm{att}_{\perp}>1.5$ ), samples (thickness $>3 \mathrm{~cm}, \mathrm{MC}<30 \%$ ) and testing frequency $(\sim 10 \mathrm{GHz})$, this effect has minor impact but reduces measurement accuracy. Again, with properly applied time gating, in both parallel and perpendicular directions only the direct path is measured.

\subsection{Transmission measurement of an isotropic DUT}

The propagation of microwaves through a DUT is generally described with the complex attenuation:

$A=e^{-\gamma_{D U T} h}$

$\mathrm{h}$ denotes the thickness of the DUT. The propagation constant $\gamma$ is a complex quantity:

$\gamma=\alpha+i \beta$

$\alpha$ is the attenuation factor which considers the exponential decrease of electric field strength with increasing propagation length in the material, and $\beta$ denotes the phase factor which considers the phase shift of the electromagnetic wave propagating through the material. $A$ is called transmission coefficient. It is a complex number describing attenuation (magnitude) and phase shift (angle) of the transmitted microwaves.

However, with a real test setup (e.g. a Vector Network Analyzer (VNA), as used in this work) it is impossible to directly measure the transmission coefficient of a DUT. A calibrated setup yields complex attenuation values corresponding to defined reference planes (see Fig. 2b, c). Thus, a VNA measurement of a DUT with a plane wave as testing signal may be described with

$V N A_{D U T}=K_{1} T^{\prime} A T^{\prime \prime} K_{2}$

with $\mathrm{K}_{1}$ and $\mathrm{K}_{2}$ complex attenuations caused by the setup from signal generation to incidence on the sample and from the exit from the sample to signal measurement, respectively. $T^{\prime}$ and $T^{\prime \prime}$ account for the above discussed reflections by only considering the wave that passes through the DUT/ air-interfaces:

$$
\begin{aligned}
& T^{\prime}=\underbrace{\frac{2}{1+\sqrt{\varepsilon_{r}}}}_{t_{\text {air } \rightarrow \text { DUT }}} \sqrt{\operatorname{Re}\left\{\sqrt{\varepsilon_{r}}\right\}} \\
& T^{\prime \prime}=\underbrace{\frac{2 \sqrt{\varepsilon_{r}}}{\sqrt{\varepsilon_{r}}+1}}_{t_{\text {DUT } \rightarrow \text { air }}} \sqrt{\operatorname{Re}\left\{\frac{1}{\sqrt{\varepsilon_{r}}}\right\}}
\end{aligned}
$$

$t_{\text {air } \rightarrow D U T}$ and $t_{D U T \rightarrow \text { air }}$ are the respective transmission factors at perpendicular incidence. The root terms constitute scaling factors between field quantities and normalized VNAmeasurements. $\varepsilon_{r}$ is part of the complex dielectric constant that describes the dielectric properties of the DUT as a lossy medium:

$\varepsilon=\varepsilon_{0} \varepsilon_{r}$

with $\varepsilon_{0}$ the permittivity of vacuum and $\varepsilon_{r}$ the relative dielectric constant

$\varepsilon_{r}=\varepsilon^{\prime}-j \varepsilon^{\prime \prime}=\varepsilon^{\prime}\left(1-j \frac{\varepsilon^{\prime \prime}}{\varepsilon^{\prime}}\right)=\varepsilon^{\prime}(1-j \cdot \tan \delta)$

$\varepsilon^{\prime}$ and $\varepsilon^{\prime \prime}$ are the real and imaginary part of the relative dielectric constant, $\tan \delta$ the loss factor. Propagation constant and the complex dielectric constant are associated by the following identity (Kark 2006):

$\gamma=\alpha+i \beta=j \omega \sqrt{\mu \varepsilon}=j 2 \pi f \sqrt{\mu_{0} \mu_{r} \varepsilon_{0} \varepsilon_{r}}$

$\mathrm{f}$ is the frequency, $\mu_{0}$ the permeability of vacuum and the relative permeability $\mu_{\mathrm{r}}=1$ for wood.

Similar to a DUT, a measurement solely through air (Fig. 2b) is described by

$V N A_{\text {air }}=K_{1} e^{-\gamma_{a i r} h} K_{2}$

The dielectric properties of air at microwave frequencies are sufficiently approximated with those of vacuum. Thus, a plane wave travelling through air features no attenuation but a phase shift depending on its frequency:

$\gamma_{\text {air }}=j \beta_{\text {air }}$

$\beta_{\text {air }}=2 \pi \frac{f}{c_{0}}$

where $f$ denotes the frequency and $c_{0}$ the speed of light in vacuum. 
Thus, with a reference measurement through air, the complex attenuations $\mathrm{K}_{1}$ and $\mathrm{K}_{2}$ caused by the measurement setup are compensated:

$C=\frac{V N A_{D U T}}{V N A_{\text {air }}}=\frac{K_{1} T^{\prime} A T^{\prime \prime} K_{2}}{K_{1} e^{-\gamma_{a i r} h} K_{2}}=T^{\prime} A e^{\gamma_{a i r} h} T^{\prime \prime}=T^{\prime} e^{-\left(\gamma_{D U T}-\gamma_{a i r}\right) h} T^{\prime \prime}$

Left and right part of Eq. (12) constitute an implicit form of a conditional equation for $\varepsilon_{\mathrm{r}}$. With $C$ the ratio of two complex attenuation measurements and Eqs. (4), (5), (8), (10) and (11) the relative dielectric constant $\varepsilon_{\mathrm{r}}$ is solved numerically with an appropriate tool, for example MATLAB. It is emphasized that the complex forms of $T^{\prime}$ and $T^{\prime \prime}$ imply that a wave transmitted through a lossy DUT encounters additional phase shifts at both air/DUTinterfaces that are not compensating each other.

$C=\underbrace{T^{\prime} T^{\prime \prime}}_{\arg ()=0.08^{\circ} \ldots 1.5^{\circ}} \underbrace{e^{-\left(\gamma_{D U T}-\gamma_{\text {air }}\right) h}}_{\arg ()=66^{\circ} \ldots 368^{\circ}}$

For the samples tested in this work, Eq. (13) shows that the contribution of $T^{\prime}$ and $T^{\prime \prime}$ to the measured phase shift could easily be neglected. In each case, a direct numerical solution of the complex $\varepsilon_{r}$ in Eq. (12) yields unstable results. Therefore, an iterative method was used. In a first step, an initial $\beta$ from the phase shift of the measured $C$ is derived. With the identity in Eq. (8), the problem of the complex $\varepsilon_{r}$ is thus reduced to a real variable for numerical solving. To account for the additional phase shift at both interfaces, the argument of the $T^{\prime} T^{\prime \prime}$-term is calculated with the obtained amplitude-corrected $\varepsilon_{r}$. The initial $\beta$ is corrected with these interface phase shifts. In a second iteration, the amplitude- and phase-corrected $\varepsilon_{r}$ is obtained.

With the derived $\varepsilon_{r}$ the VNA-ratio in Eq. (12) is corrected and the "relative" transmission coefficient obtained:

$B=A e^{\gamma_{a i r} h}=e^{-\left(\gamma_{D U T}-\gamma_{a i r}\right) h}=\frac{V N A_{D U T}}{V N A_{\text {air }}} \frac{1}{T^{\prime} T^{\prime \prime}}=C \frac{1}{T^{\prime} T^{\prime \prime}}$

With Eq. (10) this "relative" transmission coefficient describes the absolute attenuation of the DUT and the phase shift due to the DUT relative to air. Principally, the transmission coefficient of the DUT, $A$ in Eq. (1), is the physically relevant quantity. In Eq. (14) this would mean considering the exponential $\gamma_{\text {air }}$-term on the right side of equation. However, regressions to derive physical properties of a DUT that use $A$ as predictor show the demand of an additional constant term that compensates for the phase shift that is caused by an ideal lossless medium-vacuum or air, respectively. In contrast, regressions simplify by using predictors based on attenuation and phase shift exceedingly caused by the DUT rather than by an ideal, lossless medium-which is exactly this relative transmission coefficient $B$ in Eq. (14).

The work in this paper revealed that a correction of the measured VNA-ratio in Eq. (14) does not yield any noticeable improvement in precision of the derivation of physical properties. Obviously, for the chosen setup the influence of $T^{\prime}$ and $T^{\prime \prime}$ is directly proportional to the complex attenuation $A$ of the DUT. This might justify the simplification of neglecting $T^{\prime}$ and $T^{\prime \prime}$, that was applied in every former work in the field (initially Schajer and Orhan 2005), which interprets $C$ from Eq. (12) as "effective" transmission coefficient. As in this case no explicit calculation of the dielectric constant is necessary, this "effective" transmission coefficient directly serves as predictor for the derivation of desired wood physical properties.

With the use of time gating, applied in this work, and a plane wave with perpendicular incidence as testing signal, Eq. (12) is not only an idealized estimation but fully valid. Without time gating, reflections of the antennas will deteriorate measurement quality, as discussed above. However, a derivation for the transmission coefficient, for example the $T^{\prime} A T^{\prime \prime}$-term in Eqs. (3) and (12), due to multiple reflections in the DUT is given, for example, in Kark (2006).

\subsection{Transmission measurement of wood}

For wood as DUT Eq. (12) is not sufficient and a more general mathematical formulation is needed. The orthotropic nature of wood is described with two propagation constants $\gamma_{\|}$and $\gamma_{\perp}$ parallel to the grain and perpendicular to the grain, respectively.

$\left(\begin{array}{cc}C_{\|\rightarrow\|} & 0 \\ 0 & C_{\perp \rightarrow \perp}\end{array}\right)=\left(\begin{array}{cc}T_{\|}^{\prime} B_{\|} T_{\|}^{\prime \prime} & 0 \\ 0 & T_{\perp}^{\prime} B_{\perp} T_{\perp}^{\prime \prime}\end{array}\right)$

As in Eqs. (4) and (5) $T_{\|}^{\prime}$ and $T_{\|}^{\prime \prime}$ feature an $\varepsilon_{r, \|}$ and $T_{\perp}^{\prime}$ and $T_{\perp}^{\prime \prime}$ feature an $\varepsilon_{r, \perp}$.

In the general case, the grain is not aligned with the polarization of the antennas but rotated at an angle $\theta$ (Fig. 3). This is mathematically described by.

$\left(\begin{array}{cc}C_{H \rightarrow H} & C_{H \rightarrow V} \\ C_{V \rightarrow H} & C_{V \rightarrow V}\end{array}\right)=\mathbf{R}^{T}\left(\begin{array}{cc}T_{\|}^{\prime} B_{\|} T_{\|}^{\prime \prime} & 0 \\ 0 & T_{\perp}^{\prime} B_{\perp} T_{\perp}^{\prime \prime}\end{array}\right) \mathbf{R}$

with $\mathrm{R}$ the rotation matrix of the form

$\mathbf{R}=\left(\begin{array}{cc}\cos \theta & \sin \theta \\ -\sin \theta & \cos \theta\end{array}\right)$

Now, subscripts " $\mathrm{H}$ " and "V" refer to the orthogonal polarization of the measurement system (Fig. 3). The used antennas feature a cross-polarization of $<-31.3 \mathrm{~dB}$ and can 


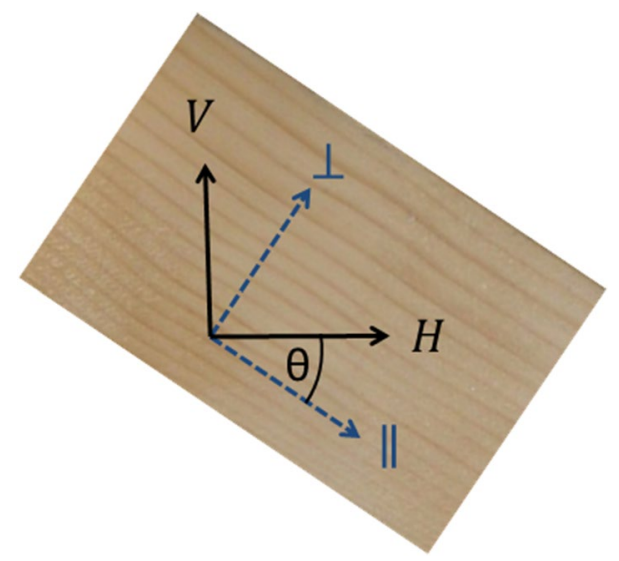

Fig. 3 Orientation of the polarization plane "V" and "H" of the testing signal and the angle $\theta$ relative to the grain direction

therefore be neglected. Thus, only a correction of attenuation and phase shift, as discussed for Eq. (12), is needed. The chosen setup (see "Materials and methods") comprises two axially aligned, mechanically rotated, linear polarized antennas. Consequently, each $C$-term on the left side of Eq. (16) constitutes a ratio of $V N A_{D U T}$ for each polarization combination to $V N A_{\text {air }}$, obtained from an air measurement with aligned polarization planes of the antennas.

The matrix-equation (16) has the typical form of an eigenvalue problem with

$\mathbf{C}=\left(\begin{array}{ll}C_{H \rightarrow H} & C_{H \rightarrow V} \\ C_{V \rightarrow H} & C_{V \rightarrow V}\end{array}\right)=\mathbf{R}^{T}\left(\begin{array}{cc}C_{\|,\|} & 0 \\ 0 & C_{\perp, \perp}\end{array}\right) \mathbf{R}$

The eigenvalues of $\mathrm{C}$ correspond to $C_{\|,\|}$and $C_{\perp, \perp}$, respectively. With Eq. (15) this yields a set of two conditional equations in the form of Eq. (12). Following the same procedure as shown for an isotropic DUT $B_{\|}$and $B_{\perp}$ are derived. The corresponding eigenvectors constitute the rotation matrix, and therefore by implication the angle of grain direction $(\theta)$, relative to the orientation of polarization plane "H" (Fig. 3).

Due to the indistinguishability of the two eigenvalues, they have to be assigned to $C_{\|,\|}$and $C_{\perp, \perp}$, respectively. This is typically carried out by assigning in relation to attenuation, with $C_{\|,\|}$the more attenuated transmission coefficient. As discussed above, it is theoretically physically possible that due to multiple reflections the measured attenuation perpendicular to the grain exceeds parallel attenuation. For wood and $10 \mathrm{GHz}$ testing frequency this would imply thin $(\sim 1 \mathrm{~cm})$, moist samples. In this rare case, the method could yield grain angles with a $90^{\circ}$-error. However, this is a physical shortcoming that holds for every approach reported (see "Introduction") although it was not discussed there.

An assignment of the respective eigenvalues to $C_{\|,\|}$ and $C_{\perp, \perp}$ via phase-shift is equally suitable, with the higher phase shift corresponding to $C_{\|,\|}$. This is suitable, as long as phase shift does not exceed $360^{\circ}$. To identify phase shifts beyond $360^{\circ}$ the typical ratio of phase shift in vs. perpendicular to grain reported by Schajer and Orhan (2006) and Aichholzer et al. (2013) can be used. For measurements at two frequencies, or in case of a multi-frequency-measurement, Trabelsi et al. (2000) showed a solution for phase ambiguity.

Note that an explicit calculation of the right side of Eq. (16) shows that both off-diagonal elements are the same. The physical interpretation is that $C_{H \rightarrow V}=C_{V \rightarrow H}$. This can also be derived from geometrical considerations. Moreover, it directly follows from the principle of reciprocity of the measurement setup. Reciprocity means that source and receiving antennas may be exchanged with each other in a measurement but still yielding the same measured values. Thus, principally only $C_{H \rightarrow V}$ or $C_{V \rightarrow H}$ has to be measured. However, measuring and considering both values in the calculation exhibits an averaging effect. Similarly, both eigenvectors yield a grain angle value. If both are considered, this implies an averaging effect for the determination of the grain direction.

Exceeding previous work, Eq. (16) now constitutes an expedient mathematical formulation for the discussed phenomena in Fig. 1, including the consideration of reflections, for the chosen setup.

\subsection{Derivation and modeling of wood physical properties}

The grain angle $\theta$ can be determined directly by the procedure described above for a single specimen. However, density and moisture content can only be derived by a statistical relationship based on measurements of several specimens.

Attenuation and phase shift of a specimen are both linearly dependent on dry density and moisture density (Torgovnikov 1993). Moisture density $D_{m}$ is defined as moisture mass per unit volume of the specimen. Dry density $D_{d}$ is defined as dry wood mass per unit volume of the specimen. In a reversal approach (Schajer and Orhan 2005), $D_{d}$ and $D_{m}$ can be estimated in a generalized form

$D_{d}=a_{1} \cdot \frac{1}{h} \cdot a t t_{d B}+a_{2} \cdot \frac{1}{h} \cdot \phi$

$D_{m}=\left(b_{1} \cdot \frac{1}{h} \cdot a t t_{d B}+b_{2} \cdot \frac{1}{h} \cdot \phi\right)\left(1+b_{3} T\right)$

$a_{1}, a_{2}, b_{1}, b_{2}$, and $b_{3}$ are the respective regression coefficients, $\mathrm{T}$ the temperature in ${ }^{\circ} \mathrm{C}$. As shown in Schajer and Orhan (2006) and Aichholzer et al. (2013), $D_{d}$ is not susceptible to temperature in the desired range of wood manufacturing $\left(\sim 4-30^{\circ} \mathrm{C}\right)$. Therefore, no temperature-related 
term is needed in Eq. (19). The mean attenuation, $a_{d B}$ (in $\mathrm{dB}$ ) and the mean phase shift, $\phi$ (in radians) are derived from

$\operatorname{att}_{d B}=20 \log _{10}\left(\frac{\left|B_{\|}\right|+\left|B_{\perp}\right|}{2}\right)$

$\phi=\frac{\operatorname{phase}\left(B_{\|}\right)+\operatorname{phase}\left(B_{\perp}\right)}{2}$

phase $\left(B_{\|}\right)$and phase $\left(B_{\perp}\right)$ are the respective unwrapped phases of $B_{\|}$and $B_{\perp}$, for example the respective arguments but corrected by multiples of $360^{\circ}$ if necessary. Both, $B_{\|}$ and $B_{\perp}$ for each measured specimen contribute to mean attenuation and mean phase shift, which has an averaging effect and uses only a minimum of variables, which improves the numerical stability.

Note that geometrical considerations suggest to only apply $B_{\perp}$ in Eqs. (21) and (22). It is clearly understood that the measurement represents a projection of the threedimensional wood fiber gradient in the plane perpendicular to the incident testing signal. With the chosen setup this projection plane coincides with the planed surface of the samples. Thus, in the case of diving grain the derived $B_{\|}$is actually a combination of $B_{\perp}$ and $B_{\|}$. The actual impact on the results will be discussed at the end of the "Results and discussion" chapter.

Obviously, Eq. (20) only holds for moist samples, as an oven-dry specimen with $D_{m}=0$ still features both attenuation and phase shift. Moreover, assumption of linearity of Eqs. (19) and (20) is only valid within a specific moisture content range with the same physical process of inclusion of water in the wood fiber. For spruce, this means moisture contents from $5 \%$ to below fiber-saturation-point, i.e. about 27\% (Torgovnikov 1993).

Density, i.e. gross density, of wood is defined as the sum of moisture density and dry density

$D=D_{m}+D_{d}$

The moisture content, $M C$, of wood is defined as the ratio of moisture and dry density

$M C=\frac{D_{m}}{D_{d}}$

The density-independent evaluation of moisture content for dielectric materials is reviewed in Nelson et al. (2001). The applicability to wood was demonstrated by Schajer and Orhan (2006). An improved equation is proposed that allows to include oven-dry samples into the regression

$M C=\left(c_{1} \frac{a t t_{d B}}{\phi}+c_{2}\right)\left(1+c_{3} T\right)$ as the additional constant term $c_{2}$ compensates for the $a t t_{d B} /$ $\varphi$-ratio of oven-dry wood. This ratio features a non-linear behavior over moisture. Thus, a quadratic equation is proposed to model non-linearity:

$M C=\left(d_{1}\left(\frac{a t t_{d B}}{\phi}\right)^{2}+d_{2} \frac{a t t_{d B}}{\phi}+d_{3}\right)\left(1+d_{4} T\right)$

Menke and Knöchel (1996) discussed and demonstrated a superior, density- and thickness-independent method to determine moisture content of tobacco by using frequency swept signals:

$X=\frac{\frac{\Delta a t_{d B}(\omega)}{\Delta \omega}}{\frac{\Delta \phi(\omega)}{\Delta \omega}}=\frac{1}{N-1} \sum_{n=1}^{N-1} \frac{a t t_{d B}\left(\omega_{n+1}\right)-a t t_{d B}\left(\omega_{n}\right)}{\phi\left(\omega_{n+1}\right)-\phi\left(\omega_{n}\right)}$

$\omega$ is the (angular) frequency, $N$ the number of tested frequencies. Menke and Knöchel (1996) provide no explicit form of how they exactly derive $X$. Therefore, the right side of Eq. (27) represents the calculation routine. With $a t t_{d B}$ from Eq. (21) and $\varphi$ from Eq. (22) this method is applied to wood. The parameter $X$ is a single value derived from the measurement of one specimen at all tested frequencies. It requires a linear behavior of the dielectric properties of the specimen over the tested frequency range. Moisture content is derived with a regression applicable to both moist and dry samples

$M C=\left(e_{1} X^{2}+e_{2} X+e_{3}\right)\left(1+e_{4} T\right)$

with the additional temperature-sensitive term in parenthesis expanding the form reported by Menke and Knöchel.

Based on Menke and Knöchel, Zhang and Okamura (1999) proposed the determination of $M C$ using phase shift measurements at two frequencies. They apply a quadratic regression, which is expanded by the parameter temperature to the form

$M C=\left(f_{1}\left(\frac{\phi_{2}}{\phi_{1}}\right)^{2}+f_{2} \frac{\phi_{2}}{\phi_{1}}+f_{3}\right)\left(1+f_{4} T\right)$

with $\varphi_{2}$ and $\varphi_{1}$ the phase shift at higher and lower frequency, respectively.

The relative dielectric constant has already been determined for each specimen applying Eqs. (4), (5), and (12). To combine all derived values in a general formulation of $\varepsilon^{\prime}, \varepsilon^{\prime \prime}$, and $\tan \delta$ as a function of moisture density, dry density, temperature and frequency, a nonlinear model was used in the form of

$\varepsilon^{\prime}=1+\left(\left(g_{1} D_{m}+g_{2} D_{d}\right)\left(1+g_{3} f\right)\left(1+g_{4} T\right)\right)$

$\varepsilon^{\prime \prime}=\left(h_{1} D_{m}+h_{2} D_{d}\right)\left(1+h_{3} f\right)\left(1+h_{4} T\right)$ 
$\operatorname{tg} \delta=\left(i_{1} D_{m}+i_{2} D_{d}\right)\left(1+i_{3} f\right)\left(1+i_{4} T\right)$

with $g_{i}, h_{i}$, and $i_{i}$ the respective fit parameters, $f$ the frequency in $\mathrm{GHz}$, and $T$ the temperature in ${ }^{\circ} \mathrm{C}$. The additional constant term in Eq. (30) is based on the same considerations as the discussion on $B$ vs. $A$ (see Eq. (14) and following). To evaluate these equations for specific values of moisture content and density refer to Eqs. (23) and (24). A discrete evaluation of the dielectric properties for moist and oven-dry samples, respectively, promises an even better model. For moist samples, Eqs. (30)-(32) apply similarly. For oven-dry wood, Eqs. (30)-(32) simplify to equations without $D_{m}$-term. In this case, $D_{d, o v e n-d r y}$ becomes identical to density of the oven-dry samples.

\section{Materials and methods}

Spruce (Picea abies (L.) Karst.) was chosen as species of interest due to its prominent role in wood processing. This is particularly true for the glue-laminated timber production in Europe. To overcome influences of local anatomical peculiarities, the wood originated from different industrial sources that had already been stored for several weeks after the drying process. The moisture content of the artificially dried wood was preliminarily evaluated using a handheld moisture meter with stick-in electrodes. Then, 87 samples were cut to $170 \mathrm{~mm} \times 170 \mathrm{~mm}$ width and $40 \mathrm{~mm}$ thickness. Care was taken to avoid any defects in the samples like knots, resin pockets, mould, or compression wood. The dimensions and mass of each sample were measured, and the density was calculated.

To obtain significant results, the sample set had to vary over the entire possible range of density and moisture content. First, the large number of samples ensures variety and the applicability of statistical methods. Furthermore, density distribution was guaranteed by randomly chosen samples. Moisture content range was defined by the production standard for glue-laminated timber in Europe [EN 386 (2001)]. It specifies minimum and maximum values of 8 and $15 \%$, respectively. This moisture content range should be covered in the sample set. Thus, several samples were conditioned again. Change in preliminarily determined moisture content was derived gravimetrically. Dimensions were re-measured and the final density, i.e. the actual density of the measured moist samples, was calculated. All samples were stored individually in hermetically-sealed plastic bags. This method assured constant moisture in the sample.

Three series of experiments were conducted at 10, 20, and $30^{\circ} \mathrm{C}$, respectively, with the moist samples, corresponding to the relevant temperature range of lamellas in glue-laminated timber fabrication. In each case, the whole
87 samples were tempered at least $24 \mathrm{~h}$ in a temperature chamber prior to the tests. Several thermocouple-equipped dummy samples were used to validate the spatial temperature distribution in the chamber.

After the measurements, the actual moisture content of the moist samples was determined with the drying method, for example gravimetrically. With the known gross density and Eqs. (23) and (24), moisture density and dry density were derived. The now oven-dried samples were subjected to the same preparation and measurement procedure again: The dimension and weight of each oven-dry specimen were determined, which yielded the density. In this case, the density is identical with the dry density $D_{d}$ of the ovendry samples. Again, samples were stored individually in hermetically-sealed plastic bags and another three series of experiments were conducted at 10,20 , and $30^{\circ} \mathrm{C}$, respectively, with the oven-dry samples.

It is a physical fact that the theoretical resolution of measurement is limited to half of the wavelength. Consequently, Torgovnikov (1993) clearly argues that with microwave testing at around $10 \mathrm{GHz}$, which corresponds to a wavelength of $30 \mathrm{~mm}$, defect-free wood may be considered as homogenous material. This work focuses on a demonstration of achievable measurement quality. For exactly this reason care was taken to prepare virtually perfect wood samples as described above. For an even more homogenous measurement, an antenna-setup was chosen that allows to evenly test a preferably big part of the entire specimen as equably as possible.

The design of the measurement system is schematically drawn in Fig. 4.

It consists of two opposed linearly polarized horn antennas, coaxial cables, and a vector network analyzer (VNA). The lens-corrected horn antennas ("Dorado LA-10-1") have a circular aperture with a diameter of $130 \mathrm{~mm}$ and a frequency range from 8 to $12.5 \mathrm{GHz}$. As was ascertained on an antenna test facility, they feature a smooth footprint on the sample even under near-field conditions, with only a slight amplification of the center area. Antenna fixtures with a stepping motor allow separate rotation of both antennas in the range from $0^{\circ}$ to $180^{\circ}$. Measurement control and further signal processing is done with a computer. For exact matching of the two polarization planes a null-finding routine in orthogonal position of the antennas is used. The setup features a measured cross-polarization suppression of $>31.3 \mathrm{~dB}$. Time gating was applied to mask undesired reflections of the antenna setup and for measuring the direct path only. The measurement position of the specimen is on a sample table between the two antennas with a distance of $30 \mathrm{~mm}$ to each antenna's aperture. The short distance from antenna aperture to specimen is beneficial to avoid diffraction at the edge of the specimen. However, the derivations in the theory chapter base on the assumption of plane 


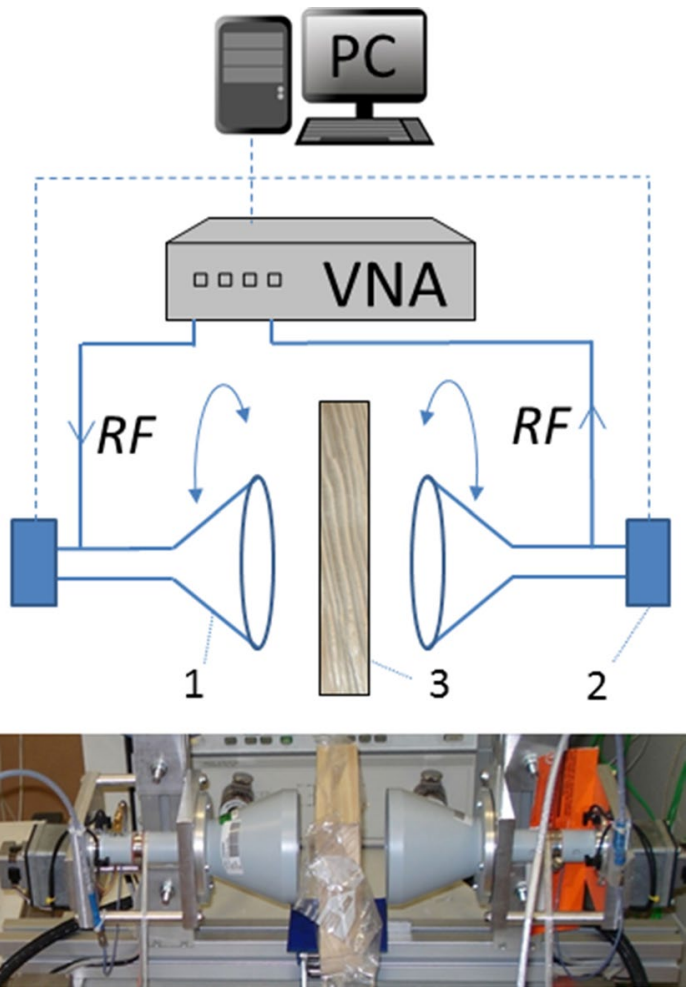

Fig. 4 Picture and schematic diagram of the microwave system: Two opposed linearly polarized horn antennas (1), coaxial cables, and a vector network analyzer (VNA) for signal processing. Antenna fixtures with stepping motors (2). Sample (3). Measurement control and further data processing with a computer $(\mathrm{PC})$

waves, which is truly valid only in far field conditions, i.e. with the present measurement setup a sample-antenna distance of more than $500 \mathrm{~mm}$. Thus, measurements presented in this paper are performed in near field conditions. Nonetheless, the Results chapter will prove the theoretical derivations are sufficiently approximated with the chosen setup.

In each measurement a frequency sweep is performed in 51 steps from 8 to $12 \mathrm{GHz}$ yielding the necessary data for the evaluation of the dielectric constant. Multiple testing spread across a wide frequency range is a prerequisite for time gating and moisture evaluation with Eqs. (28) and (29).

First, a reference measurement is carried out to compensate for the frequency response of the measurement setup yielding the respective $V N A_{\text {air }}$-value for Eq. (15).

Subsequently, the specimens (still wrapped in hermetically sealed plastic bags) are inserted, and four measurements are performed with each specimen with an orthogonal antenna setup, yielding the respective $V N A_{D U T}$-values for Eq. (15) and allowing the calculation of grain angle, $B_{\perp}$ and $B_{\|}$. The whole procedure is repeated with $30^{\circ}, 60^{\circ}$, and $90^{\circ}$ rotation of the coordinate system of the measurement, making it possible to prove the method is equally suitable

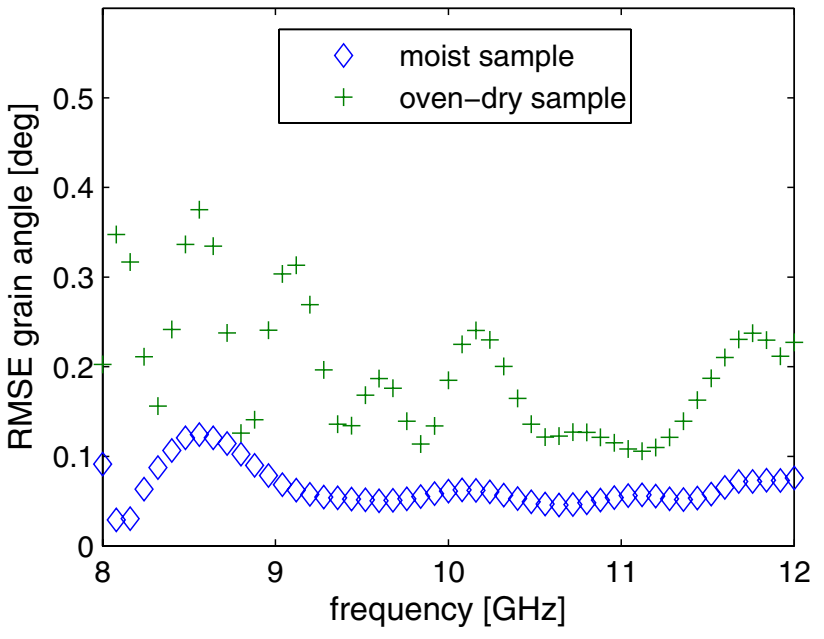

Fig. 5 Typical standard errors for grain angle measurements from 8 to $12 \mathrm{GHz}$ for one single specimen

over the entire possible range of grain angle deviation. Multiple measurements on one specimen at different orientations are a prerequisite for the error estimation of grain angle measurement.

For the estimation of error, the root-mean-squared error (RMSE) is consistently used in this work:

$R M S E=\sqrt{\frac{1}{n} \sum_{i=1}^{n}\left(y_{i}-\hat{y}_{i}\right)^{2}}$

with $y_{i}$ the estimated values and $\hat{y}_{i}$ the actual values.

\section{Results and discussion}

Grain angle detection is presented first. This is followed by the derivation of dry density and moisture density for each of the 51 frequencies. Subsequently, moisture content estimation using single frequency signals and frequency swept signals, respectively, is shown. Finally, the evaluation and modelling of the dielectric constant is presented. The chapter is closed with general discussions.

\subsection{Grain angle}

The quality of grain angle detection is exemplarily shown in Fig. 5 for one specimen. As it is impossible to obtain exact grain angles from the sample for comparison with measured values, following Schajer and Orhan (2006) another approach is chosen. Due to the highly precise antenna positioning, grain angle values derived at different antenna positions are compared, which is performed separately for each of the 51 measured frequencies. Hence, 
the derived error is a measure for the reproducibility of the grain angle measurement at a specific frequency. RMSE over the entire frequency range from 8 to $12 \mathrm{GHz}$ is maximum $0.14^{\circ}$ for moist samples and $0.40^{\circ}$ for oven-dry samples. The undulating trend of the RMSE over frequency is a characteristic of the applied time gating. The magnitude of the error for oven-dry samples is mainly caused by an unexpected variation of the attenuation values in the measurements. Obviously, oven-dry samples show a stronger impact of near-field effects of the antennas. Still, even the oven-dry samples feature superior quality of grain angle detection compared to other work in the field.

\subsection{Dry density}

Dry density estimation (measured range from 284 to $527 \mathrm{~kg} / \mathrm{m}^{3}$ ) for both moist and oven-dry samples, respectively, was performed for each of the 51 measured frequencies. The respective estimation errors are shown in Fig. 6. It was also tried to apply the derived regression to $D_{d, \text { moist (samples) }}$ to estimate $D_{d, d r y \text { (samples). The }}$ results show very similar standard deviation like the other $\mathrm{D}_{\mathrm{d}}$-estimations $\left(12.5 \mathrm{~kg} / \mathrm{m}^{3}\right)$ but are heavily biased, featuring a RMSE of $48 \mathrm{~kg} / \mathrm{m}^{3}$, demonstrating that Eq. (19) for $D_{d, \text { moist }}$ is limited to moisture contents of 5-27\%, where the assumption of linearity is reasonable (as discussed in the "Theory" chapter). However, it still covers the entire moisture content range relevant for the production of glued laminated timber.

Estimations for $D_{d, d r y}$ (RMSE maximum $12.4 \mathrm{~kg} / \mathrm{m}^{3}$ ) feature similar RMSE to $D_{d, \text { moist }}$ (maximum $11.5 \mathrm{~kg} / \mathrm{m}^{3}$ ). However, the devolution of $a_{i, o v e n-d r y}$ over frequency shows that the regression is compensating for the encountered variation in oven-dry attenuation measurement. Therefore, an estimation of $D_{d, \text { oven-dry }}$ exclusively with the dominant quantity for oven-dry samples, phase shift was additionally

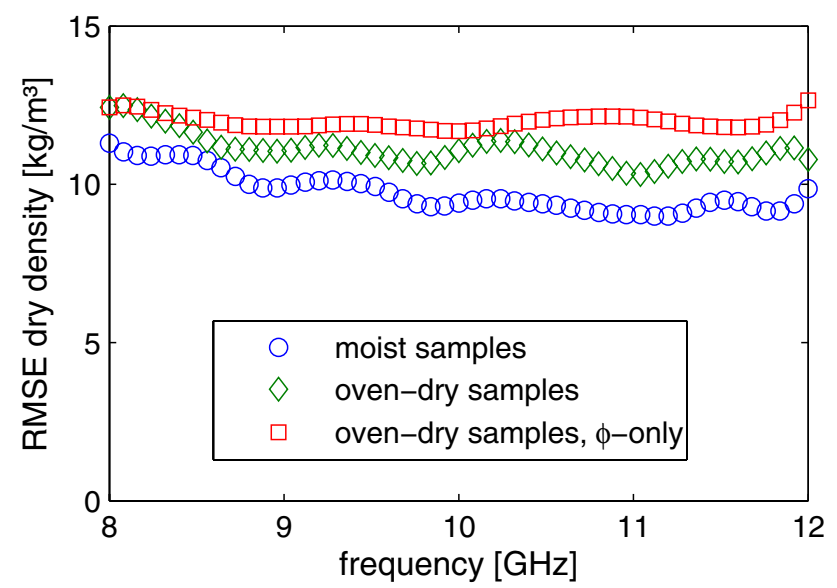

Fig. 6 RMSE for dry density estimation from 8 to $12 \mathrm{GHz}$ performed. The regression yielded one single coefficient $a_{2, \text { oven-dry, } \phi-o n l y}$ that shows a smooth behavior over frequency and a similar RMSE of maximum $12.6 \mathrm{~kg} / \mathrm{m}^{3}$ (Fig. 6).

\subsection{Moisture density}

The estimation of moisture density $D_{m}$ (measured range from 27 to $56 \mathrm{~kg} / \mathrm{m}^{3}$ ) was performed for each of the 51 measured frequencies. The respective RMSE are given in Fig. 7 with a maximum of $1.8 \mathrm{~kg} / \mathrm{m}^{3}$.

As for dry density, due to the same physical processes and consequential linearity, these results can be extrapolated for spruce samples with moisture contents from 5 to $27 \%$.

\subsection{Moisture content (single frequency)}

An explicit calculation of moisture content with Eq. (24) yields an error of about $1 \%$. The direct evaluation of moisture content with a density-independent approach in Eq. (25) yields superior results (presented in Figs. 8, 9). Tested range spans from $0 \%$ (both oven-dry and moist samples) or $7.6 \%$ (moist samples only) to $14 \%$. Figure 8 a shows estimated vs. actual moisture content at $10 \mathrm{GHz}$ for regression exclusively with moist samples. Figure 9 shows the RMSE at the respective frequency with a maximum of $0.45 \%$. The use of an additional constant parameter $c_{2}$ is justified as it compensates for the $a t t_{d b} / \phi$-ratio of oven-dry wood. The improvement manifests not only in a smaller estimation error but also an even distribution in Fig. 8a. Prior work (Aichholzer et al. 2013) showed a moisture content distribution slightly inclined to the ideal trend line and an estimation error of maximum $0.47 \%$. As discussed, these results can be extrapolated for spruce samples with moisture contents from 5 to $27 \%$.

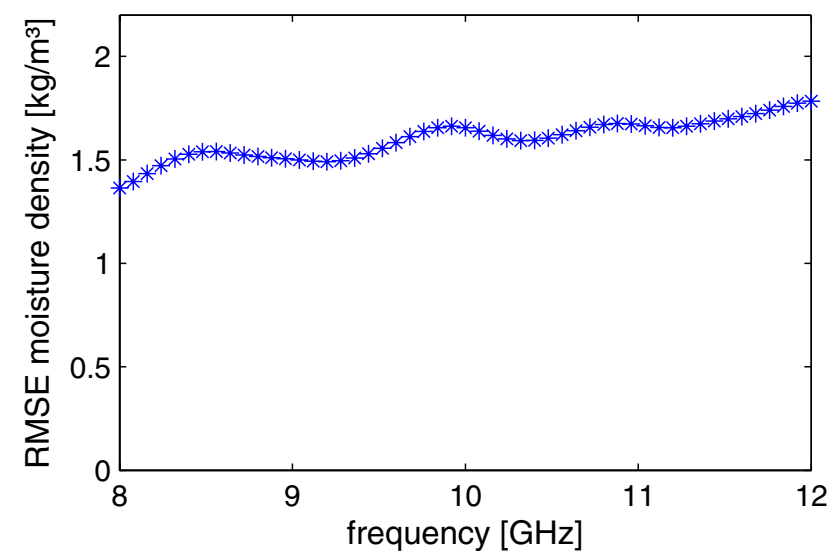

Fig. 7 RMSE for moisture density estimation from 8 to $12 \mathrm{GHz}$ 

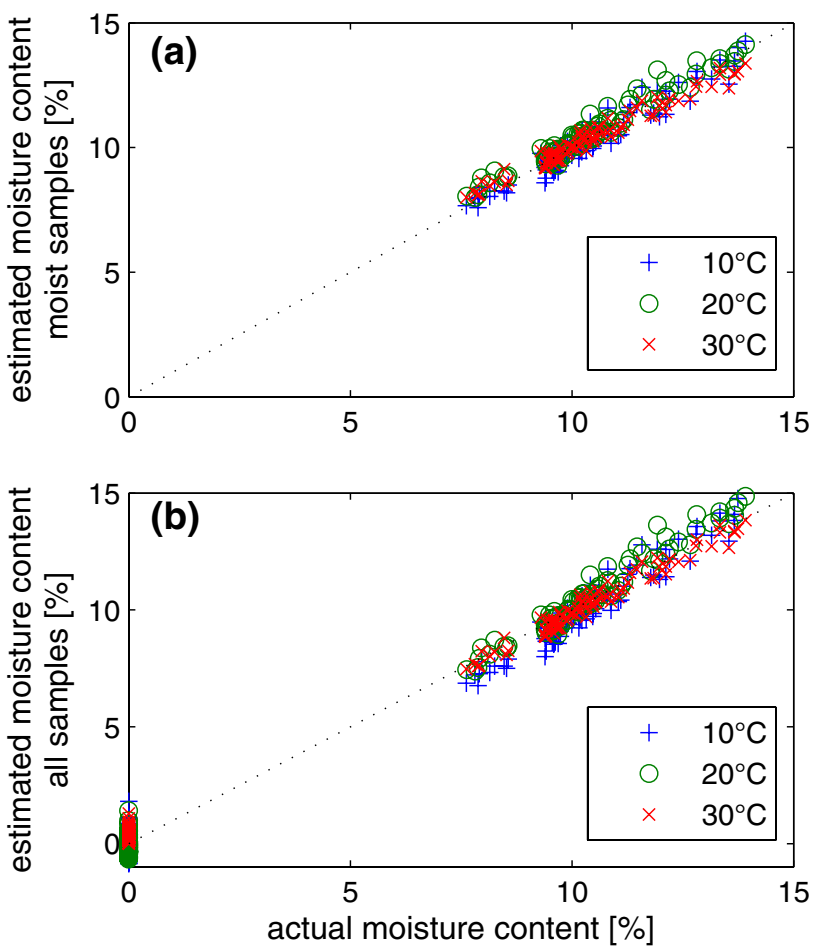

Fig. 8 Estimated vs. actual moisture content at $10 \mathrm{GHz}$ : a moist samples $\mathbf{b}$ all, i.e. moist and oven-dry, samples

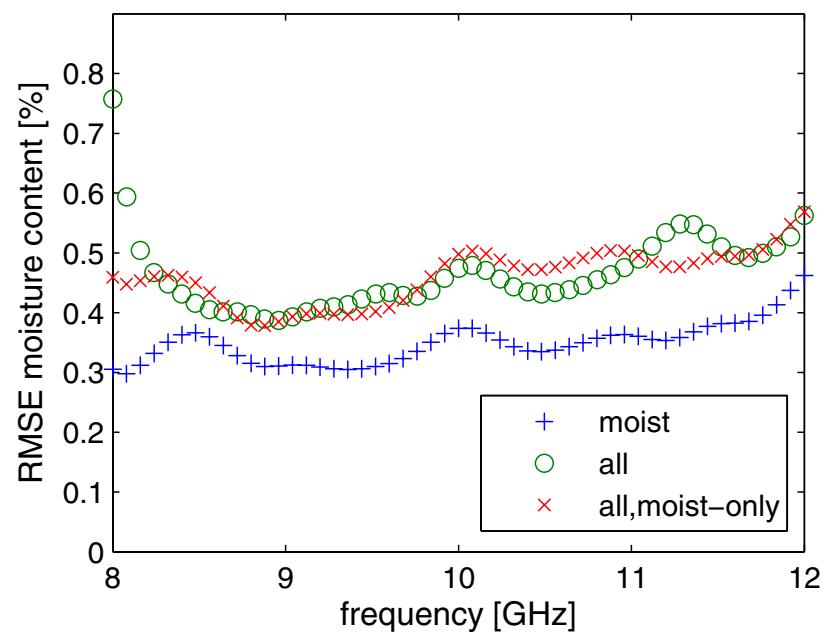

Fig. 9 RMSE for moisture content estimation at a single frequency

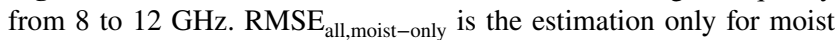
samples but based on the regression for all, i.e. both moist and ovendry, samples

Including oven-dry samples in the regression Eq. (25) an extension of detectable moisture content range is proposed. Figure $8 \mathrm{~b}$ shows the estimated vs. actual moisture content at $10 \mathrm{GHz}$ for regression with all, i.e. both moist and oven-dry samples. The distribution of the moist samples is slightly deteriorated, the estimation error
(RMSE ${ }_{\text {all }}$ in Fig. 9) slightly higher. However, with a typical RMSE of smaller than $0.6 \%$ it still proves reasonable to describe both dry and moist samples in one regression model. The estimation error of only the moist samples, based on the regression Eq. (25) derived for all samples, $\mathrm{RMSE}_{\text {all,moist-only }}$ is maximum $0.55 \%$ (Fig. 9). All regression coefficients feature a smooth devolution over frequency. A quadratic regression term, as shown in Eq. (26), yielded no remarkable changes for the estimation of moist samples. However, for the regression with all samples, RMSE was noticeably better but the additional term scattered heavily due to the variations in oven-dry measurements and was therefore omitted.

\subsection{Moisture content (frequency sweep)}

Moisture content estimation with Eq. (28) is based on frequency swept signals and yields superior results. Again, tested range spans from $0 \%$ (oven-dry and moist samples) or $7.6 \%$ (moist samples only) to $14 \%$. For each frequency sweep from 8 to $12 \mathrm{GHz}$ one single estimation parameter $X$ [from Eq. (27)] is derived. Figure 10 shows the parameter $X$ plotted vs. the actual moisture content. For the estimation of moist samples, exclusively, a simple and robust model with only a linear term and temperature compensation is sufficient (dotted lines). With the coefficients $e_{2}=-422.3, e_{4}=-0.0092$, and $e_{1}=e_{3}=0$ it yields an RMSE of $0.32 \%$. Figure 11a compares the estimated with actual moisture content values for moist samples. Again, this result may be extrapolated for spruce samples with moisture contents from 5 to $27 \%$. For an entire

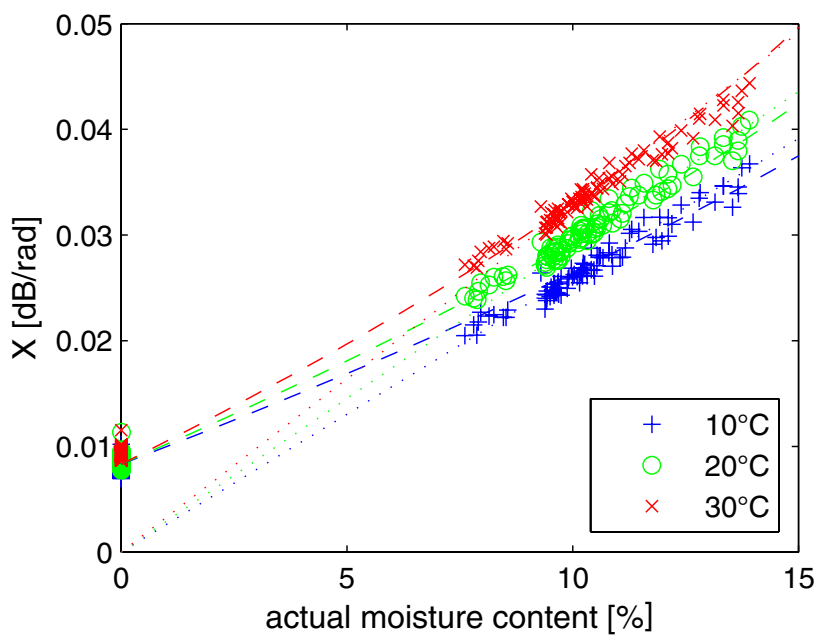

Fig. 10 Fit parameter $X$ (derived from frequency swept signal) at different temperatures vs. actual moisture content. The linear regression solely for moist samples (dotted) and the more sophisticated regression for all samples (dashed) is drawn for all temperatures 

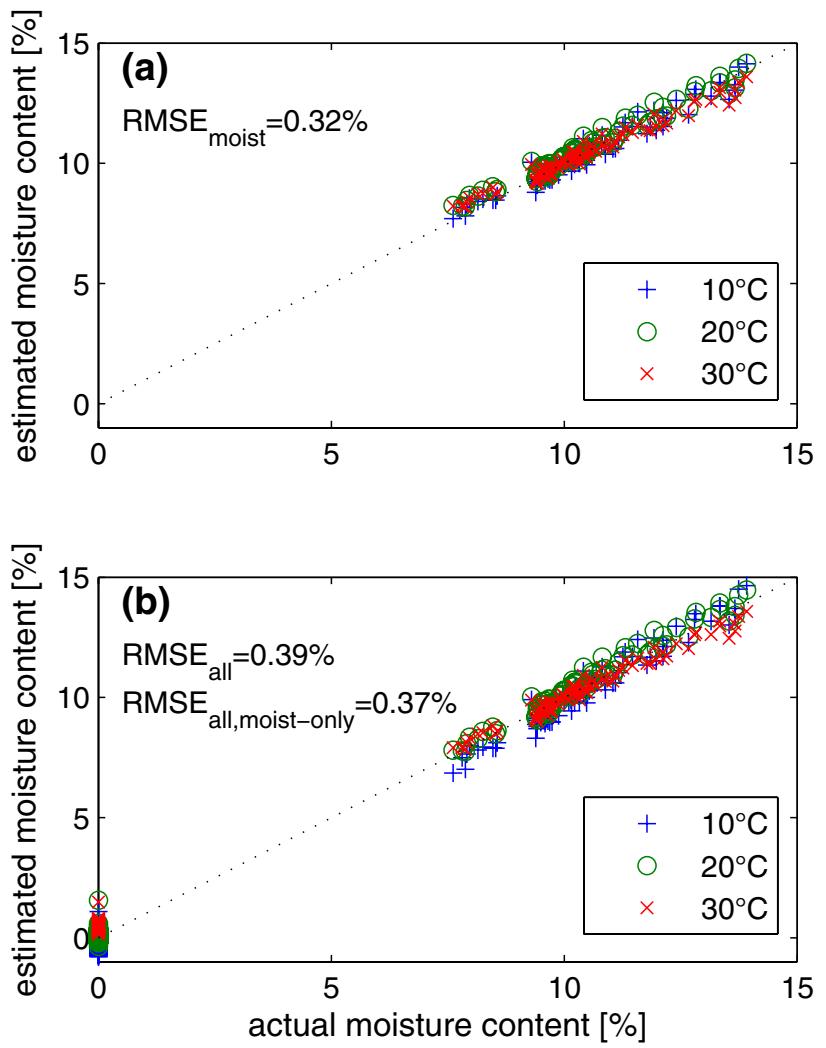

Fig. 11 Estimated vs. actual properties of moisture content of the frequency swept method. a Linear regression solely for moist samples. b Non-linear regression for all, i.e. both moist and oven-dry, samples

description of all, i.e. both moist and oven-dry, samples all five coefficients of Eq. (28) are needed. The corresponding regression for all samples is plotted in Fig. 10 (dashed lines). Regression coefficients for all samples are derived with $e_{1}=-3751, e_{2}=746.3, e_{3}=-5.275$, $e_{4}=-0.0105$. Figure $11 \mathrm{~b}$ shows the estimated vs. actual moisture contents. The estimation error for all samples is $\mathrm{RMSE}_{\text {all }}=0.39 \%$. The estimation error only for moist samples, based on the regression for all samples, is $\mathrm{RMSE}_{\text {all,moist-only }}=0.37 \%$. Following Menke and Knöchel (1996) this method is assumed to apply not only to moisture contents up to fiber-saturation-point but even beyond. In addition, based on frequency swept data it exhibits an intrinsic averaging effect. Phase measurement is improved by phase tracking vs. frequency in contrast to estimating integral phase through a layer of wood, which is prone to phase ambiguity. Even without an applied time gating, the calculation of $X$ in Eq. (27) compensates for notches in the frequency response (erroneous measurement at single frequencies due to multiple reflections). Moreover, such single erroneous measurements are revealed by irregularities in the devolution of the $a t t_{d B} / \phi$ ratio over frequency and may selectively be discarded.
This ability simultaneously allows for improved grain angle detection via selecting, and averaging over, only properly measured values. Thus, even without time-gating but suitable measures to weaken undesired antenna reflections (as discussed in the "Theory" chapter), this method is expected to yield very similar results for moisture content and grain angle detection as presented in this work.

Moisture estimation following Zhang and Okamura (Eq. 29) is based on the ratio of phase shift measurements at two different frequencies. However, this ratio features rather strong variation for similar actual moisture contents, including the oven-dry samples. Hence, a moisture content estimation based on all samples yields an $\mathrm{RMSE}_{\text {all }}=1.50 \%$. This value is derived for $\phi_{1}$ at $8.6 \mathrm{GHz}$ and $\phi_{2}$ at $11.4 \mathrm{GHz}$, but it does not change significantly with other frequencies. Considering only moist samples and a linear regression, i.e. $f_{1}=0$ in Eq. (29), an RMSE $\mathrm{moist}=0.70 \%$ is derived. In this case, the use of a quadratic term in Eq. (29) for moist samples slightly improves the quality of the regression but shows strange behavior at lower moisture content. Obviously, samples with a wide spread moisture content spectrum are demanded for a robust regression. Therefore, this method is inferior to other procedures presented to evaluate moisture content. However, similar results to Zhang and Okamura are reported, but in consideration of the additional regression parameter temperature.

\subsection{Dielectric constant}

Equations (30)-(32) describe the dielectric constant for spruce in respect to tested moisture density, dry density, temperature and frequency. The derived coefficients for the respective conditional equation are given in Table 1 . The encountered variations in some of the attenuation measurements of oven-dry wood resulted in sporadic values very close to zero for $\varepsilon_{\perp, \text { oven-dry }}^{\prime \prime}$ and $\tan \delta_{\perp, \text { oven-dry. }}$

The fit of the dielectric constants is linear in frequency. A quadratic term did not yield any advantage and was therefore omitted, which also justifies the application of Eq. (27) for the use of frequency-swept data, which implies linear dependency on frequency in the tested range. A discrete evaluation of moist and oven-dry samples featured slightly better estimation errors but tended to model measurement uncertainties and yielded unphysical behavior (positive frequency coefficient for $\varepsilon_{\|, \text {oven-dry }}^{\prime \prime}$ and negative $D_{d}$ coefficient for $\varepsilon_{\|, \text {moist }}^{\prime \prime}$ and $\varepsilon_{\perp, \text { moist }}^{\prime \prime}$. Thus, one single equation is preferably used to describe one dielectric constant for all tested samples.

Using Eqs. (23) and (24) dielectric constant values for moisture content of 0 and $10 \%$, density of 300 
Table 1 Derived dielectric constants of spruce

\begin{tabular}{|c|c|c|c|c|c|c|c|c|}
\hline \multirow{2}{*}{$\begin{array}{l}\text { Spruce (MC } \\
0-14 \%)\end{array}$} & \multirow[t]{2}{*}{ Equations } & \multirow[t]{2}{*}{ Coeff. } & \multicolumn{4}{|c|}{ Coefficient index i } & \multirow[t]{2}{*}{ Range of values } & \multirow[t]{2}{*}{ Regression, RMSE } \\
\hline & & & 1 & 2 & 3 & 4 & & \\
\hline$\varepsilon_{\|}^{\prime}$ & (30) & $\mathrm{g}_{\|, \mathrm{i}}$ & 0.0109 & 0.0019 & -0.0103 & 0.0057 & $1.53-2.75$ & 0.0419 \\
\hline$\varepsilon_{\|}^{\prime \prime}$ & (31) & $\mathrm{h}_{\|, \mathrm{i}}$ & 0.0052 & $9.410 \times 10^{-5}$ & -0.0152 & 0.0296 & $0.021-0.673$ & 0.0262 \\
\hline $\operatorname{tg} \delta_{\|}$ & (32) & $\mathrm{i}_{\|, \mathrm{i}}$ & 0.0023 & $5.706 \times 10^{-5}$ & -0.0081 & 0.0212 & $0.012-0.263$ & 0.0093 \\
\hline$\varepsilon_{\perp}^{\prime}$ & $(30)$ & $\mathrm{g}_{\perp, \mathrm{i}}$ & 0.0067 & 0.0013 & -0.0072 & 0.0041 & $1.36-2.23$ & 0.0318 \\
\hline$\varepsilon_{\perp}^{\prime \prime}$ & (31) & $\mathrm{h}_{\perp, \mathrm{i}}$ & 0.0023 & $2.941 \times 10^{-5}$ & -0.0184 & 0.0288 & $0.000 *-0.282$ & 0.0128 \\
\hline $\operatorname{tg} \delta_{\perp}$ & (32) & $\mathrm{i}_{\perp, \mathrm{i}}$ & 0.0013 & $2.029 \times 10^{-5}$ & -0.015 & 0.0236 & $0.000 *-0.133$ & 0.0051 \\
\hline
\end{tabular}

Coefficients of the respective Eqs. (1) and (2) for a general description. The range of values and RMSE of the non-linear fit

*These values are discussed in the "Results and discussion" chapter

Table 2 Dielectric constants from regressions (30)-(32) at $\mathrm{T}=20^{\circ}$, $\mathrm{f}=10 \mathrm{GHz}$, and moisture content 0 and $10 \%$, respectively, compared to data reported from other authors (Torgovnikov 1993)

\begin{tabular}{|c|c|c|c|c|}
\hline & \multicolumn{2}{|c|}{$D=300 \mathrm{~kg} / \mathrm{m}^{3}$} & \multicolumn{2}{|c|}{$D=500 \mathrm{~kg} / \mathrm{m}^{3}$} \\
\hline & This work & $\begin{array}{l}\text { Torgovnikov } \\
\text { (1993) }\end{array}$ & This work & $\begin{array}{l}\text { Tor- } \\
\text { govnikov } \\
(1993)\end{array}$ \\
\hline \multicolumn{5}{|c|}{$\mathrm{T}=20^{\circ} \mathrm{C}, \mathrm{f}=10 \mathrm{GHz}, M C=10 \%$} \\
\hline$\varepsilon_{\|}^{\prime}$ & 1.80 & 1.86 & 2.34 & 2.32 \\
\hline$\varepsilon_{\|}^{\prime \prime}$ & 0.23 & 0.14 & 0.38 & 0.31 \\
\hline $\operatorname{tg} \delta_{\|}$ & 0.102 & 0.075 & 0.171 & 0.135 \\
\hline$\varepsilon_{\perp}^{\prime}$ & 1.54 & 1.6 & 1.90 & 2.0 \\
\hline$\varepsilon_{\perp}^{\prime \prime}$ & 0.090 & 0.08 & 0.15 & 0.18 \\
\hline $\operatorname{tg} \delta_{\perp}$ & 0.050 & 0.05 & 0.083 & 0.09 \\
\hline \multicolumn{5}{|c|}{$\mathrm{T}=20^{\circ} \mathrm{C}, \mathrm{f}=10 \mathrm{GHz}, M C=0 \%$} \\
\hline$\varepsilon_{\|}^{\prime}$ & 1.56 & 1.62 & 1.93 & 1.97 \\
\hline$\varepsilon_{\|}^{\prime \prime}$ & 0.038 & 0.034 & 0.064 & 0.065 \\
\hline $\operatorname{tg} \delta_{\|}$ & 0.022 & 0.021 & 0.037 & 0.033 \\
\hline$\varepsilon_{\perp}^{\prime}$ & 1.39 & 1.4 & 1.65 & 1.7 \\
\hline$\varepsilon_{\perp}^{\prime \prime}$ & 0.011 & 0.020 & 0.019 & 0.037 \\
\hline $\operatorname{tg} \delta_{\perp}$ & 0.008 & 0.014 & 0.013 & 0.022 \\
\hline
\end{tabular}

and $500 \mathrm{~kg} / \mathrm{m}^{3}$, temperature of $20^{\circ} \mathrm{C}$, and frequency of $10 \mathrm{GHz}$ are derived and compared to reported values from Torgovnikov (1993) in Table 2. Small variations are expected, as wood is not homogeneous but a natural, heterogeneous material. Thus, Eqs. (30)-(32) with the parameters from Table 1 generate a full set of dielectric constants a that is applicable to simulation and modelling of spruce in respect of moisture density (or moisture content), dry density (or density), frequency $(8-12 \mathrm{GHz})$, and temperature $\left(10-30^{\circ} \mathrm{C}\right)$.

\section{Discussion}

As proposed in the "Theory" chapter, all regressions for densities and moisture content were additionally performed only with $B_{\perp}$ as predictor. All results deteriorated by about $20 \%$. Obviously, the impact of averaging over both relative transmission coefficients $B_{\|}$and $B_{\perp}$ supersedes the geometrical uncertainty of $B_{\|}$, at least for the samples tested in this work. Thus, for typical diving grain values $\left(<10^{\circ}\right)$ the averaging over $B_{\|}$and $B_{\perp}$ turns out to be a superior predictor of the heterogeneous wood anatomy.

As already pointed out in the "Theory" chapter, an evaluation based on $C$ as predictor yielded virtually the same results. This interpretation of $C$ as "effective" transmission coefficient avoids the corrections in Eq. (14) and the explicit calculation of $\varepsilon_{\mathrm{r}}$, which simplifies the evaluation. It is emphasized that this finding is only valid for the presented setup with time gating and not necessarily expandable to other setups with unconsidered or undefined reflections.

Throughout this work in all regressions with temperature an additional quadratic temperature term was tested but yielded no improvement of the regressions. Furthermore, no influence of the antenna position, i.e. orientation of the polarization plane of the incident signal, relative to the grain direction, could be detected in any of the evaluations emphasizing that the method is equally suitable for all possible grain directions. 


\section{Implementation}

The scope of this work was a feasibility study. Consequently, the chosen measurement setup featured laboratory style equipment. For industrial applications, this equipment is too expensive, unsuited for the targeted environment, and has a too large noise figure. While the latter is not a problem for classical VNA measurements, it requires strong averaging if very low power levels are used, as required by EMC/emission-directives. Using a VNA therefore requires strong averaging, resulting in long measurement times.

An industrial implementation is highly specified for its very purpose and features the same accuracy as a laboratory setup. The rotating antennas of the setup in this work shall be replaced by dual-polarized antennas with electronic polarization control. Such antennas allow switching between both polarization planes within a few nanoseconds. Doing a one-time calibration of the antennas impairments and applying the calibration to measurements, the setup yields the same accuracy as mechanically rotated single polarized antennas. By further using low noise amplifiers, the noise level can be dramatically reduced, which translates into higher measurement speeds of a dedicated hardware compared to a VNA. A quantitative example for such a built-up is given in Leder (2012) where a low-cost, noise optimized setup features $>100,000$ single frequency measurements per second. Contemporary progress of circuitry-wise know-how and hardware even allows considerably more performance. Frequency swept measurements are reasonable with about 1000 measurements per second. However, as the limiting factor on measurement speed, signal-to-noise-ratio (SNR), scales with transmitting power, higher magnitudes of measurements per second are achievable. While this amplified testing signal would potentially require a housing fitted with noise suppression, the amplitude would still be in the milliwatt-range. Thus, it is emphasized that even an amplified testing signal constitutes no harm for men and no shielding measures, as mandatory for X-ray-based scanners, for example, are needed.

More localized measurements are also possible, for example, by application of the modulated scattering technique (Schajer and Orhan 2005), a focused beam setup (Bogosanovic et al. 2013), or a localized antenna array (Denzler et al. 2013). However, as pointed out in Materials and Methods, the theoretical resolution of measurement is physically limited to half of the wavelength. Moreover, actual applications realistically feature resolution limits of one or one-and-a-half wavelength. Edge diffraction, especially for small timber dimensions, is a physical fact and design issue. Its deteriorating impact on measurement quality can be addressed by appropriate antenna and setup design as well as by advanced mathematical routines. This is a scope of future work.

\section{Conclusion}

A detailed discussion of free space measurement demonstrated the requirement of a dual-linear-polarized measurement setup and emphasized the need of coping with reflections in wood measurements. A prototype system meeting this demand is used to determine key physical data of moist and oven-dry spruce [Picea abies (L.) Karst.]. Grain angle is detected with a RMSE of $0.14^{\circ}$ (moist) and $0.4^{\circ}$ (ovendry) in the entire possible range from $-90^{\circ}$ to $+90^{\circ}$. The RMSE of dry density is maximum $11.5 \mathrm{~kg} / \mathrm{m}^{3}$ (moist) and $12.6 \mathrm{~kg} / \mathrm{m}^{3}$ (oven-dry) with measured range from 284 to $527 \mathrm{~kg} / \mathrm{m}^{3}$. Moisture density estimation features a maximum RMSE of $1.8 \mathrm{~kg} / \mathrm{m}^{3}$ (range $27-56 \mathrm{~kg} / \mathrm{m}^{3}$ ). Adapted regression models for density- and thickness-independent moisture content evaluation at a single frequency are proposed. They yield superior results to prior work for moist samples with a RMSE of $0.44 \%$ (measured range 7.6-14\%) and allow the inclusion of oven-dry wood in the moisture estimation (RMSE $0.57 \%$, range 0-14\%). With frequency swept moisture measurements, all samples are described with one regression featuring a RMSE of $0.39 \%$ (range 0-14\%). A moisture evaluation based on phase shifts at two frequencies only yielded inferior results with RMSE of $0.7 \%$ and above. All moisture evaluations yield a very similar, frequency independent and modest dependency on temperature. The idealized orthotropic wood model proves applicable to microwave testing of wood.

The presented calculation procedure for transmission coefficient and grain angle exceeds previous approaches by considering the occurring reflections and taking full benefit of the matrix algebra. Furthermore, it paves way for a mathematical formulation and modelling of multiple reflections leading to implicit forms of transmission coefficients which allow a deeper evaluation and understanding and a more general discussion of Free-Space transmission measurement of wood, for example non-perpendicular incidence of testing signal. This will be the scope of future work.

The advantage of frequency swept measurements was discussed and demonstrated. Based on the results of Menke and Knöchel (1996), this procedure should also be applicable to moisture contents beyond the fiber-saturation point. Even without an applied time gating, this method is capable to cope with the effects due to multiple reflections in the DUT. Thus, a measurement setup with several testing frequencies can provide superior moisture measurement and simultaneously improve quality of grain angle detection. Such a measurement setup is feasible with today's progress of circuit design and measurement technology. With the fast and continuous improvement and availability of the electronic hardware and antenna design, even industrial low-cost applications with time-gating are reasonable in the near future. 
Acknowledgements Open access funding provided by TU Wien (TUW). This work is part of a PhD-thesis supported by the University of Natural Resources and Life Sciences, Vienna, and the Vienna University of Technology, Vienna. Experimental work was carried out, with support of our project partner MiCROTEC GmbH—slr, within the framework of the "Industrielles Kompetenzzentrum Holztechnologie", financially supported by the Austrian Federal Ministry of Economics and Labour, the Association of the Austrian Wood Industries, and the Austrian Federal Forests.

Open Access This article is distributed under the terms of the Creative Commons Attribution 4.0 International License (http:// creativecommons.org/licenses/by/4.0/), which permits unrestricted use, distribution, and reproduction in any medium, provided you give appropriate credit to the original author(s) and the source, provide a link to the Creative Commons license, and indicate if changes were made.

\section{References}

Agilent Technologies (2007) Time domain analysis using a network analyzer. Application Note 1287-12. Agilent Technologies

Aichholzer A, Arthaber H, Schuberth C, Mayer H (2013) Non-destructive evaluation of grain angle, moisture content and density of spruce with microwaves. Eur J Wood Prod 71:779-786

Al-Mattarneh HMA, Ghodgaonkar DK, Majid WMWA (2001) Microwave nondestructive testing for classification of Malaysian timber using free-space techniques. In: Signal processing and its applications, sixth international, symposium, vol 2, pp 450-453

Bogosanovic M, Al-Anbuky A, Emms G (2011). Microwave measurement of wood anisotropy. In: 2011 IEEE sensors applications symposium (SAS), pp 262-267

Bogosanovic M, Al-Anbuky A, Emms GW (2013) Microwave nondestructive testing of wood anisotropy and scatter. IEEE Sens J 13:306-313

Denzler JK, Weidenhiller A (2015) Microwave scanning as an additional grading principle for sawn timber. Eur J Wood Prod 73:423-431

Denzler JK, Koppensteiner J, Arthaber H (2013) Grain angle detection on local scale using microwave transmission. Int Wood Prod J 4:68-74

Denzler JK, Lux C, Arthaber H (2014) Contactless moisture content and density evaluation of sawn timber using microwave transmission. Int Wood Prod J 5:200-206

EN 1912 (2012) Structural timber. Strength classes. Assignment of visual grades and species. Eur. Comm. Stand

EN 386 (2001) Glued laminated timber-performance requirements and minimum production requirements. Eur. Comm. Stand

Heikkila S, Jakkula P, Tiuri M (1982) Microwave methods for strength grading of timber and for automatic edging of boards. In: Microwave conference, 12th European, pp 599-603

James WL, Yen Y-H, King RJ (1985) A microwave method for measuring moisture content, density, and grain angle of wood. Research Note FPL-0250, pp 1-9

Johansson J, Hagman O, Fjellner B-A (2003) Predicting moisture content and density distribution of Scots pine by microwave scanning of sawn timber. J Wood Sci 49:312-316

Kark K (2006) Antennen und Strahlungsfelder (Vieweg, Germany) (Antennas and radiation fields) (In German). 2nd edn (ISBN 13 978-3-8348-0216-3)

King RJ, Yen YH (1981) Probing amplitude, phase, and polarization of microwave field distributions in real time. IEEE Trans Microw Theory Tech MTT 29:1225-1231

Kollmann FFP, Côté WA (1984) Solid wood. Springer, Berlin

Kraszewski A, Trabelsi S, Nelson S (2001) Broadband microwave wheat permittivity measurements in free space. J Microw Power Electromagn Energy Publ Int Microw Power Inst 37:41-54
Leder N (2012). X-band transmission analyzer with multi antenna control. Diploma thesis. TU, Wien

Leicester RH, Seath CA (1996) Application of microwave scanners for stress grading. In: Conference proceedings, international wood engineering conference, $\mathrm{pp}$ 435-440

Lundgren N, Hagman O, Johansson J (2006) Predicting moisture content and density distribution of Scots pine by microwave scanning of sawn timber II: evaluation of models generated on a pixel level. J Wood Sci 52:39-43

Malik SA, Ghodgaonkar DK, Hambaly AMBA, Majid WMWA, Nuruddin MF (2005) Measurement of wood grain angle using free-space microwave measurement system in $8-12 \mathrm{GHz}$ frequency range. In: 2005 Asian conference on sensors and the international conference on new techniques in pharmaceutical and biomedical research, pp 213-218

Martin P, Collet R, Barthelemy P, Roussy G (1987) Evaluation of wood characteristics: internal scanning of the material by microwaves. Wood Sci Technol 21:361-371

Menke F, Knochel R (1996) New density-independent moisture measurement methods using frequency-swept microwave transmission. In: Microwave Symposium Digest, IEEE MTT-S international, vol 3, pp 1415-1418

Nelson SO, Trabelsi S, Kraszewski AW (2001) RF sensing of grain and seed moisture content. IEEE Sens J 1:119

Nyström J (2003) Automatic measurement of fiber orientation in softwoods by using the tracheid effect. Dev Image Process Scanning Wood 41:91-99

Robertson SH, Buckmaster HA (1992) A laboratory experiment to determine the complex refractive index of layered dielectrics at 9 GHz. Am J Phys 60:933-937

Schajer GS (2001) Lumber strength grading using X-ray scanning. For Prod J 51:43-50

Schajer GS, Orhan FB (2005) Microwave non-destructive testing of wood and similar orthotropic materials. Subsurf Sens Technol Appl 6:293-313

Schajer GS, Orhan FB (2006) Measurement of wood grain angle, moisture content and density using microwaves. Holz Roh Werkst 64:483-490

Shen J, Schajer G, Parker R (1994) Theory and practice in measuring wood grain angle using microwaves. IEEE Trans Instrum Meas 43:803-809

Tiuri M, Heikkila S (1979) Microwave instrument for accurate moisture measurement of timber. In: Microwave conference, 9th European, pp 702-705

Tiuri M, Liimatainen P (1974) Microwave method for measurement of fiber orientation in paper. In: Conf Proc Eur Microw Conf. $137-140$

Torgovnikov GI (1993) Dielectric properties of wood and wood-based materials. Springer, Berlin

Trabelsi S, Kraszewski AW, Nelson SO (2000) Phase-shift ambiguity in microwave dielectric properties measurements. IEEE Trans Instrum Meas 49:56-60

Vallejos C, Grote W (2009) Wood moisture content measurement at $2.45 \mathrm{GHz}$. In: Microwave and optoelectronics conference (IMOC), 2009 SBMO/IEEE MTT-S international, pp 221-225

Vikberg T, Oja J, Antti L (2012a) Moisture content measurement in scots pine by microwave and X-rays. Wood Fiber Sci 44:280-285

Vikberg T, Hansson L, Schajer GS, Oja J (2012b) Effects on microwave measurements and simulations when collecting data close to edges of wooden boards. Measurement 45:525-528

Wilson PJ (1999) Accuracy of a capacitance-type and three resistance-type pin meters for measuring wood moisture content. For Prod J 49:29-32

Zhang Y, Okamura S (1999) New density-independent moisture measurement using microwave phase shifts at two frequencies. IEEE Trans Instrum Meas 48:1208-1211 